\title{
QUASI-HOMOGENEOUS ASSOCIATIVE FUNCTIONS
}

\author{
BRUCE R. EBANKS
}

Department of Mathematics

Marshall University

Huntington, WV 25755, U. S. A.

(Received July 15, 1996 and in revied version March 18, 1997)

\begin{abstract}
A triangular norm is a special kind of associative function on the closed unit interval $[0,1]$. Triangular norms (or $t$-norms) were introduced in the context of probabilistic metric space theory, and they have found applications also in other areas, such as fuzzy set theory. We determine the explicit forms of all $t$-norms which satisfy a generalized homogeneity property called quasi-homogeneity.
\end{abstract}

KEY WORDS AND PHRASES: Triangular norm ( $t$-norm), homogeneous function, probabilistic metric space.

1991 AMS SUBJECT CLASSIFICATION CODES: 39B12, 54E70, 39B22.

\section{INTRODUCTION}

Let $I=[0,1]$ be the closed unit interval on the real line $\mathbf{R}$. A triangular norm (or $t$-norm) is a map $T: I \times I \rightarrow I$ satisfying the following four hypotheses. First, $T$ is associative:

$$
T(x, T(y, z))=T(T(x, y), z), \quad x, y, z \in I
$$

second, $T$ is nondecreasing in each variable; third, $T$ is commutative; and fourth, for all $x \in I$,

$$
T(x, 1)=x .
$$

$T$-norms were introduced by Menger [1] as a means of generalizing the triangle inequality to statistical (later, probabilistic) metric spaces. Associated with a family $\left\{F_{p q}\right\}$ of probability distribution functions is a map $T: I \times I \rightarrow I$ (later, a $t$-norm) such that

$$
\left.F_{p r}(x+y) \geq T F_{p q}(x), F_{q r}(y)\right]
$$

for all $p, q, r$ in some space $S$ and all nonnegative reals $x$ and $y$. Interpreting $F_{p q}(x)$ as the probability that the distance between $p$ and $q$ is less than $x$, inequality $(0.2)$ means that the probability that the distance from $p$ to $r$ is less than $x+y$ is at least as great as the $T$-value of the probabilities that the distance from $p$ to $q$ is less than $x$ and the distance from $q$ to $r$ is less than $y$. For further information on the history, theory and applications of $t$-norms, see Schweizer and Sklar [2].

In light of the interpretation above, we examine now those $t$-norms satisfying an additional property. If the probabilities $F_{p q}(x)$ and $F_{q r}(y)$ on the right side of $(0.2)$ are shrunk by a factor $t \in I$, then it may be reasonable to suppose that the probability $F_{p r}(x+y)$ is also shrunk by a factor depending on $t$, possibly after some change of scale. More precisely, we shall study those $t$-norms $T$ which satisfy 


$$
T(t x, t y)=\Pi^{-1}\{\varphi(t) \Pi[T(x, y)]\}
$$

for some map $\varphi: I \rightarrow I$ and some continuous injection $\Pi: I \rightarrow[0, \infty)$. Such $t$-norms will be called quasihomogeneous. If $\Pi$ in ( 0.3$)$ is the identity map, then $T$ is called homogenous, and the forms of such $t$ norms are known already (e.g. [3]). In that case, either $T(x, y)=\operatorname{Min}(x, y)$ with $\varphi(t)=t$, or $T(x, y)$ $=x y$ with $\varphi(t)=t^{2}$. We shall obtain that result as a corollary of the main result of the present paper, in which we determine the general solution of $(0.3)$ for $t$-norms.

Before proceeding, let us observe some other properties of $t$-norms. It is easy to deduce the following from the definition of triangular norm:

$$
\begin{array}{cc}
T(x, 0)=T(0, x)=0, & x \in I, \\
T(x, y) \leq \operatorname{Min}(x, y), & x, y \in I
\end{array}
$$

(i.e. Min is the "maximal" $t$-norm), so in particular

$$
T(x, x) \leq x, \quad x \in I .
$$

Note also that $(0.3)$ forces $\Pi$ to be strictly increasing. For, setting $x=y=0$ there, we have $T(0,0)=\Pi^{-1}\{\varphi(t) \Pi[T(0,0)]\}$. Since $T(0,0)=0$, this means $\Pi(0)=\varphi(t) \Pi(0)$ for all $t \in[0,1]$. Hence $\Pi(0)=0$, since otherwise $\varphi \equiv 1$, which in $(0.3)$ with $t=0$ would yield $0=T(x, y)$ for all $x, y \in$ $I$, in violation of $(0.1)$.

\section{PRELIMINARIES}

In the sequel, let $\overline{\mathbf{R}}^{+}=[0, \infty]$ be the extended nonnegative real half-line. The structure of continuous $t$-norms is known. (The continuity assumption can be weakened somewhat, but that is not relevant to the current discussion.)

THEOREM 1.1. (Schweizer and Sklar [2: Sections 5.3-5.5]) Let $T: I \times I \rightarrow I$ be a continuous $t$-norm.

(i) $T$ satisfies $T(x, x)<x$ for all $x \in(0,1)$ if and only if $T$ admits the representation

$$
T(x, y)=f[g(x)+g(y)]
$$

where $g: I \rightarrow \overline{\mathbf{R}}^{+}$is continuous, strictly decreasing with $g(1)=0$, where $\mathrm{f}: \overline{\mathbf{R}}^{+} \rightarrow I$ is onto, continuous, strictly decreasing on $[0, g(0)]$ with $f(u)=0$ for $u \geq g(0)$, and where $f \circ g$ is the identity map on $I$ (i.e. $g$ is a "quasi-inverse" of $f$ ).

(ii) If $T(x, x)=x$ for all $x \in I$, then $T=$ Min on $I \times I$.

(iii) Otherwise, the semigroup $(I, T)$ is an ordinal sum of semigroups $\left\{\left(S_{k}, T_{k}\right)\right\}$. Here each $S_{k}$ is a proper closed (nontrivial) subinterval of $I$, each $T_{k}$ admits a representation of the form (1.1) on $S_{k} \times S_{k}$, with $g_{k}$ on $S_{k}$ and $f_{k}$ onto $S_{k}$, and $T=\operatorname{Min}$ on $(I \times I) \backslash \bigcup_{k}\left(S_{k} \times S_{k}\right)$ [2].

REMARK. Any $t$-norm of the form given by case (i) of Theorem 1.1 is called Archimedean. If in addition $g(0)=\infty$, then $f=g^{-1}$ and $T$ is called strict.

We shall use Theorem 1.1 to find the forms of quasi-homogeneous $t$-norms. We shall also need the following known result.

THEOREM 1.2. (See e.g. Aczel and Dhombres [4: Chapter 15, Theorem 1]. The general solution of

$$
g(t x)=a(t) g(x)+b(t)
$$

among maps $g, a, b: I \rightarrow \overline{\mathbf{R}}^{+}$, is given by the following: 


$$
\begin{array}{r}
g(x)=\ell(x)+c, a(x)=1, b(x)=a(x) ; \\
g(x)=c, a \text { arbitrary, } b(x)=c[1-a(x)] ;
\end{array}
$$

or

$$
g(x)=c m(x)+d, a(x)=m(x), b(x)=d[1-m(x)]
$$

for all $x \in I$, where $c$ and $d$ are arbitrary constants, $\iota . I \rightarrow \overline{\mathbf{R}}^{+}$is an arbitrary solution of the logarithmic functional equation

$$
\alpha(x y)=\alpha(x)+\alpha(y), \quad x, y \in I
$$

and $m: I \rightarrow \overline{\mathbf{R}}^{+}$is an arbitrary solution of the multiplicative functional equation

$$
m(x y)=m(x) m(y), \quad x, y \in I
$$

\section{DETERMINATION OF QUASI-HOMOGENEOUS T-NORMS}

We prove first that quasi-homogeneity implies that $T$ must be continuous and that only certain forms are possible for $\varphi$.

LEMMA 2.1. If a $t$-norm $T$ is quasi-homogeneous in the sense of $(0.3)$, then $T$ is continuous and there exists a constant $\alpha>0$ such that

$$
\varphi(t)=t^{\alpha}
$$

PROOF: Setting $x=y=1$ in (0.3), we find that

$$
\Pi[T(t, t)]=\varphi(t) \Pi(1),
$$

since $T(1,1)=1$. Since $\Pi(1) \neq 0$, this yields

$$
\varphi(t)=\Pi(1)^{-1} \Pi[T(t, t)]
$$

and shows immediately that $\varphi$ is monotonic, with $\varphi(1)=1$. It shows also that $\varphi$ is multiplicative, since by (0.3) we have

$$
\begin{aligned}
\varphi(x y) & =\Pi(1)^{-1} \Pi[T(x y, x y)] \\
& =\Pi(1)^{-1} \varphi(x) \Pi[T(y, y)] \\
& =\varphi(x) \varphi(y) .
\end{aligned}
$$$$
x, y \in I \text {. }
$$

Moreover, (2.3) shows that $\varphi(t)<1$ for $t<1$, for otherwise, since $\Pi$ is strictly increasing we would have $T(t, t) \geq 1$ for some $t<1$, contradicting $T \leq$ Min. Thus $\varphi$ must be of the form (2.1) for some $\alpha>0$.

Now (0.3) takes the form

$$
T(t x, t y)=\Pi^{-1}\left\{t^{\alpha} \Pi[T(x, y)]\right\}
$$

For any given $x, y \in I$, if $x \leq y$ we have 


$$
\Pi \circ T(x, y)=\Pi \circ T\left(y \cdot \frac{x}{y}, y \cdot 1\right)=y^{\alpha} \Pi \circ T\left(\frac{x}{y}, 1\right)=y^{\alpha} \Pi\left(\frac{x}{y}\right)
$$

while if $x>y$, then

$$
\Pi \circ T(x, y)=\Pi \circ T\left(x \cdot 1, x \cdot \frac{y}{x}\right)=x^{\alpha} \Pi \circ T\left(1, \frac{y}{x}\right)=x^{\alpha} \Pi\left(\frac{y}{x}\right) .
$$

In either case, putting $m=\operatorname{Min}(x, y)$ and $M=\operatorname{Max}(x, y)$, we obtain

$$
T(x, y)=\Pi^{-1}\left\{M^{\alpha} \Pi\left(\frac{m}{M}\right)\right\} .
$$

Since $\Pi$ is continuous, this shows that $T$ is continuous and completes the proof of the lemma.

We observe in passing that the full force of the definition of $t$-norm was not used in Lemma 2.1. In fact, Lemma 2.1 is valid also whenever $T: I \times I \rightarrow I$ is quasi-homogeneous, satisfies $T(1, x)=T(x, 1)$ $=x$, and the diagonal map $t \rightarrow T(t, t)$ is non-decreasing and satisfies $T(t, t)<1$ for all $t<1$.

. Now we may assume that $T$ is continuous and use Theorem 1.1 to obtain additional information about the structure of $T$. The next step is to deal with the Archimedean case.

THEOREM 2.2. $T: I \times I \rightarrow I$ is a quasi-homogeneous $t$-norm satisfying $T(x, x)<x$ for all $x \in(0,1)$ if and only if either

$$
T(x, y)=x y,
$$

for all $x, y \in I$, with $\varphi$ given by (2.1) and with $\Pi(t)=\Pi(1) t^{\alpha / 2}$ for some $\alpha>0$, or

$$
T(x, y)= \begin{cases}\left(x^{\beta}+y^{\beta}-1\right)^{1 / \beta} & , \forall x, y \in(0,1] \\ 0 & , \text { otherwise }\end{cases}
$$

for some $\beta<0$, with $\varphi$ given by (2.1) for some $\alpha>0$ and with $\Pi(t)=\Pi(1)\left[\frac{1}{2}\left(t^{\beta}+1\right)\right]^{\alpha / \beta}$ if $t>0$ and $\Pi(0)=0$.

PROOF: Suppose $T$ is a quasi-homogeneous $t$-norm with $T(x, x)<x$ on $(0,1)$. By Lemma 2.1, $T$ is continuous and $\varphi$ has the form (2.1) for some $\alpha>0$. Thus by Theorem 1.1(i), $T$ has the form (1.1) where $g$ is a quasi-inverse of $f$. Inserting the representation (1.1) into (2.4), we get

$$
f[g(t x)+g(t y)]=\Pi^{-1}\left\{t^{\alpha} \Pi \circ f[g(x)+g(y)]\right\} .
$$

First, we establish that $g(0)=\infty$ (and hence $f=g^{-1}$ and $T$ is strict). Suppose, to the contrary, that $g(0)$ $<\infty$. Since $g$ is continuous, we can choose positive $t$ so close to 0 that $g(t)>\frac{1}{2} g(0)$. Recalling that $g(1)=0, f(0)=1$ and $f(u)=0$ for $u \geq g(0)$, we deduce from (2.7) by putting $x=y=1$ that

$$
0=f[2 g(t)]=\Pi^{-1}\left\{t^{\alpha} \Pi(1)\right\}
$$

for all $t$ sufficiently close to 0 . But this implies $\Pi(0)=0=\Pi(1)$, contradicting the injectivity of $\Pi$. Thus $g(0)=\infty$ and $f=g^{-1}$.

Now we can write (2.7) as 


$$
g(t x)+g(t y)=g \circ \Pi^{-1}\left\{t^{\alpha} \Pi \circ g^{-1}[g(x)+g(y)]\right\}
$$

where $g$ is a strictly decreasing homeomorphism of $I$ onto $\overline{\mathbf{R}}^{+}$. Fixing $t$ temporarily, let $u=g(x), v=$ $g(y)$ and define

$$
g_{t}(x):=g(t x), k_{t}(x):=g \circ \Pi^{-1}\left[t^{\alpha} \Pi(x)\right]
$$

for $x \in I$. Then (2.8) becomes

$$
g_{t} \circ g^{-1}(u)+g_{t} \circ g^{-1}(v)=k_{t} \circ g^{-1}(u+v), \quad u, v \in \overline{\mathbf{R}}^{+}
$$

Since $g_{t}$ and $k_{t}$ are strictly monotonic by definition, the solution of this Pexider equation is

$$
g_{t} \circ g^{-1}(u)=a_{t} u+b_{t}, k_{t} \circ g^{-1}(u)=a_{t} u+2 b_{t}
$$

for some "constants" $a_{t}$ and $b_{t}$ (depending on $t$ ). Freeing $t \in I$ and recalling the definitions of $g_{t}$ and $k_{t}$, we have now

$$
\begin{gathered}
g(t x)=a(t) g(x)+b(t), \\
g \circ \Pi^{-1}\left[t^{\alpha} \Pi(x)\right]=a(t) g(x)+2 b(t),
\end{gathered}
$$

valid for all $t, x \in I$.

The general solution of equation (2.9) is given in Theorem 1.2. We eliminate solution (1.4) here because $g$ is strictly monotonic. We consider solutions (1.3) and (1.5) separately.

Case 1. Suppose the solution of (2.9) is of the form (1.3). Since $g$ is strictly decreasing, so is the logarithmic function $l$. That is, there exists a constant $b<0$ for which

$$
g(x)=b \log x+c, a(t)=1, \text { and } b(t)=\log t
$$

Substituting these into (2.10), we find that

$$
t^{\alpha} \Pi(x)=\Pi\left(x t^{2}\right)
$$

With $x=1$, this yields $\Pi(q)=q^{\alpha / 2} \Pi(1)$ for all $q \in 1$. Furthermore, inserting (2.11) into (1.1), with $f$ $=g^{-1}$, we obtain (2.5) for $T$.

Case 2. Suppose the solution of (2.9) is of the form (1.5). Since $g$ is strictly monotonic, the same is true of the multiplicative function $m$, so $m(x)=x^{\beta}$. Moreover, since $g(0)=\infty$ and $g(1)=0$, we must have

$$
g(x)=c\left(x^{\beta}-1\right), a(t)=t^{\beta}, \text { and } b(t)=c\left(t^{\beta}-1\right)
$$

for some constants $\beta<0$ and $c>0$. Inserting these into (2.10) and simplifying, we arrive at

$$
\Pi^{-1}\left[t^{\alpha} \Pi(x)\right]=\left[(t x)^{\beta}+t^{\beta}-1\right]^{1 / \beta}
$$

Putting $x=1$ and $q=\left(2 t^{\beta}-1\right)^{1 / \beta}$, this gives 


$$
\Pi(q)=\Pi(1)\left[\frac{1}{2}\left(q^{\beta}+1\right)\right]^{\alpha / \beta}
$$

Finally, (2.12) combined with (1.1) (and $f=g^{-1}$ ) yields

$$
T(x, y)=\left\{1+\frac{1}{c}\left[c\left(x^{\beta}-1\right)+c\left(y^{\beta}-1\right)\right]\right\}^{1 / \beta}
$$

which simplifies to (2.6).

The converse is easily verified, and that completes the proof of Theorem 2.2 .

Now we are ready to establish the main result.

THEOREM 2.3. $T: I \times I \rightarrow I$ is a quasi-homogeneous $t$-norm if and only if $T$ is given by (2.5), by (2.6) for some $\beta<0$, or by $T(x, y)=\operatorname{Min}(x, y)$. In the last case, $\varphi$ is given by (2.1) for some $\alpha$ $>0$, and $\Pi$ has the form $\Pi(x)=\Pi(1) x^{\alpha}$.

REMARK. If we define $T_{\beta}$ to be the $t$-norm given by (2.6), then

$$
\lim _{\beta \rightarrow 0} T_{\beta}(x, y)=x y \text {, and } \lim _{\beta \rightarrow-\infty} T_{\beta}(x, y)=\operatorname{Min}(x, y) .
$$

Defining $T_{0}$ to be the product on $I \times I$ and $T_{-\infty}$ to be Min, we can restate the conclusion of Theorem 2.3 as follows. The only quasi-homogeneous $t$-norms are the members of the family $\left\{T_{\beta}\right\}$ for $-\infty \leq \beta \leq 0$.

PROOF OF THEOREM 2.3: Let $T$ be a quasi-homogeneous $t$-norm. Then by Lemma 2.1, $T$ is continuous and $\varphi$ has the form (2.1) for some $\alpha>0$. Now we apply Theorem 1.1.

If case (i) of Theorem 1.1 holds, then we find that (2.5) or (2.6) holds by Theorem 2.2.

In case (ii) of Theorem 1.1, we have $T=\mathrm{Min}$, and equations ( 0.3$)$ and (2.1) yield

$$
\operatorname{Min}(t x, t y)=\Pi^{-1}\left\{t^{\alpha} \Pi \circ \operatorname{Min}(x, y)\right\} .
$$

With $x=y=1$, we obtain

$$
\Pi(t)=t^{\alpha} \Pi(1), \quad t \in I .
$$

Finally, we consider case (iii) of Theorem 1.1. Choose any $x_{0} \in(0,1)$ such that $T\left(x_{0}, x_{0}\right)=$ $x_{0}$, and let $S_{k}$ be any subinterval of $\left[x_{0}, 1\right]$ such that $T_{k}$ admits representation (1.1) in the square $S_{k} \times S_{k}$. Then

$$
T(x, y)=\operatorname{Min}(x, y), \quad(x, y) \in D_{1} \cup D_{2},
$$

where $D_{1}$ and $D_{2}$ are the rectangles

$$
D_{1}=\left\{(x, y) \mid 0 \leq y \leq x_{0} \leq x \leq 1\right\}, D_{2}=\left\{(x, y) \mid 0 \leq x \leq x_{0} \leq y \leq 1\right\}
$$

in $I \times I$. We prove that in fact this case cannot occur.

Let us confine our attention to the lower triangle $\{(x, y) \mid 0<y \leq x \leq 1\}$. Choose $(x, y)$ in $S_{k} \times S_{k}$ so that $x_{0}<y \leq x \leq 1$. Then we choose $t=\frac{x_{0}}{y}$, so that $(t x, t y)$ belongs to $D_{1}$. Now $(0.3)$ and (2.1) yield

$$
\Pi[T(x, y)]=\Pi\left(x_{0}\right)\left(\frac{y}{x_{0}}\right)^{\alpha},
$$


which implies that $T(x, y)$ is independent of $x$ for $x \geq y>x_{0}$. Specifically,

$$
T(x, y)=\Pi^{-1}\left[\left(\frac{y}{x_{0}}\right)^{\alpha} \Pi\left(x_{0}\right)\right]
$$

when $x \geq y>x_{0}$, which is inconsistent with representation (1.1) for the restriction $T_{k}$ of $T$ to $S_{k} \times S_{k}$. Indeed, as in the proof of Theorem 2.2, $T_{k}$ must be strict:

$$
T_{k}(x, y)=g_{k}^{-1}\left[g_{k}(x)+g_{k}(y)\right], \quad x, y \in S_{k} .
$$

This representation, with strictly monotonic $g_{k}$, shows that $T_{k}(x, y)$ is injective in $x$ for each $y \in S_{k}$. But the right hand side of (2.13) is independent of $x$. This contradiction shows that there can be no $S_{k}$ in the interval $\left[x_{0}, 1\right]$.

A similar argument shows that there can be no $S_{k}$ in the interval $\left[0, x_{0}\right]$. Thus there can be no proper ordinal sum. That is, case (iii) of Theorem 1.1 is incompatible with quasi-homogeneity. (In other words, if a quasi-homogeneous $T$ satisfies $T\left(x_{0}, x_{0}\right)=x_{0}$ for some $x \in(0,1)$, then it satisfies $T(x, x)$ $=x$ for all $x \in(0,1)$.)

The converse is easily verified, and this completes the proof of the theorem.

From Theorem 2.3, the structure theorem for homogeneous $t$-norms is easily extracted.

COROLLARY 2.4. $T: I \times I \rightarrow I$ is a homogeneous $t$-norm if and only if either $T(x, y)=$ $x y$, with $\varphi(t)=t^{2}$, or $T(x, y)=\operatorname{Min}(x, y)$, with $\varphi(t)=t$.

PROOF: Suppose $T$ is a homogeneous $t$-norm. We apply Theorem 2.3 in the special case in which $\Pi$ in (0.3) is the identity map. When $T$ is of the form (2.5), we have (cf. Theorem 2.2) $\Pi(x)=$ $\Pi(1) x^{\alpha / 2}$. This will give $\Pi(x)=x$ only if $\alpha=2$; in (2.1) this yields $\varphi(t)=t^{2}$. When $T$ is given by (2.6) for some $\beta<0$, we note that $\Pi(x)=\Pi(1)\left[\frac{1}{2}\left(x^{\beta}+1\right)\right]^{\alpha / \beta}$. Such $\Pi$ can never be the identity map, so this case cannot arise. Finally, when $T(x, y)=\operatorname{Min}(x, y)$, the accompanying $\Pi$ is (cf. Theorem 2.3) $\Pi(x)=\Pi(1) x^{\alpha}$. This $\Pi$ is the identity only if $\alpha=1$, in which case $(2.1)$ becomes $\varphi(t)=t$.

The simple converse completes the proof.

\section{REFERENCES}

[1] MENGER, K. Statistical metrics, Proc. Nat. Acad. Sci. USA 28 (1942), 535-537.

[2] SCHWEIZER, B. and SKLAR, A. Probabilistic Metric Spaces, North-Holland, New York, Amsterdam, Oxford, 1983.

[3] SCHWEIZER, B. personal communication.

[4] ACZEL, J. and DHOMBRES, J. Functional Equations in Several Variables, Cambridge U. Press, Cambridge, New York, Melbourne, 1989. 


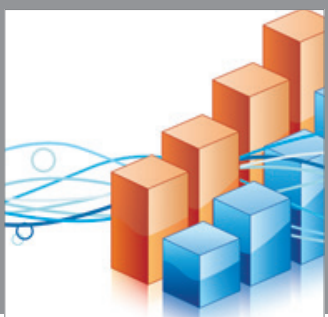

Advances in

Operations Research

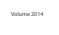

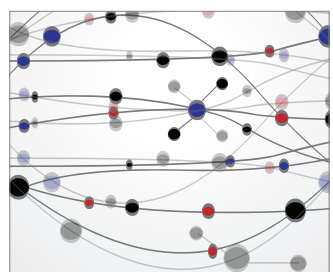

\section{The Scientific} World Journal
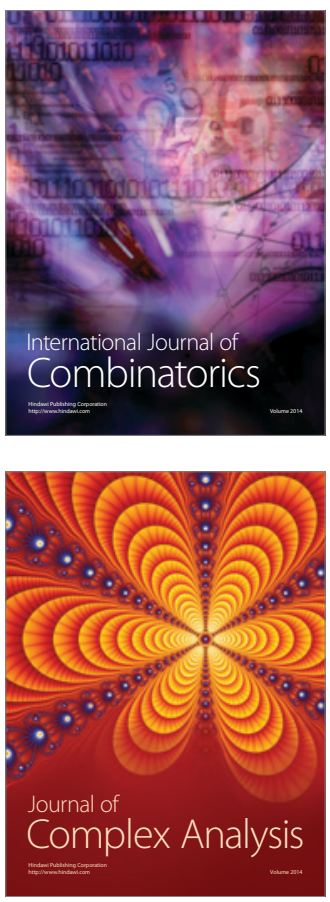

International Journal of

Mathematics and

Mathematical

Sciences
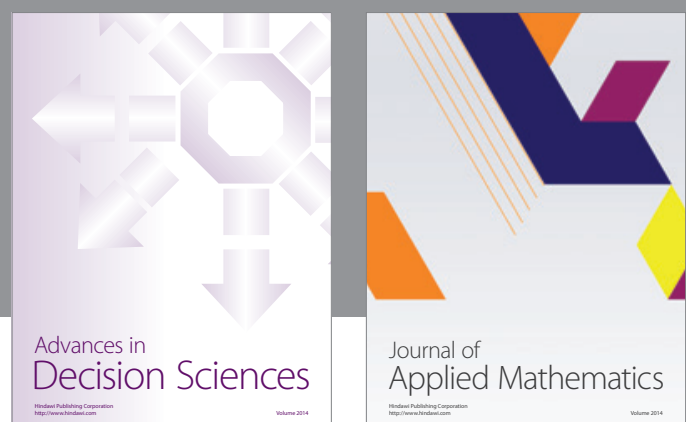

Journal of

Applied Mathematics
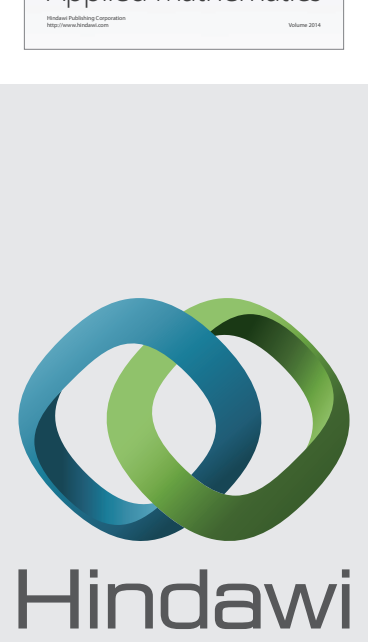

Submit your manuscripts at http://www.hindawi.com
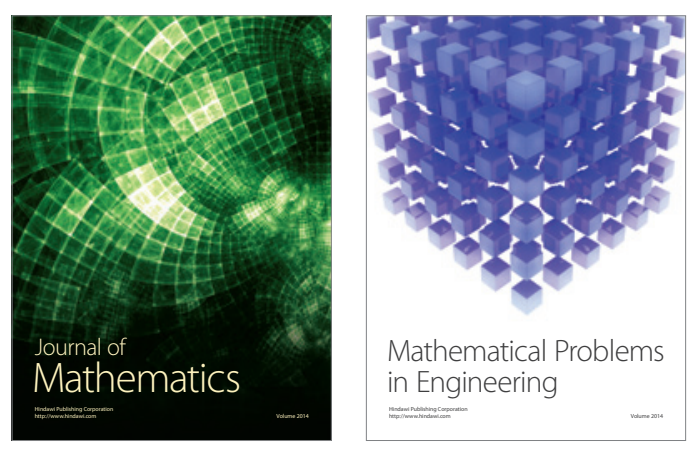

Mathematical Problems in Engineering
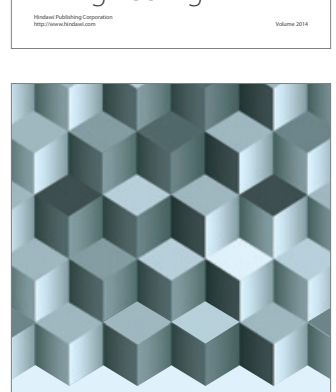

Journal of

Function Spaces
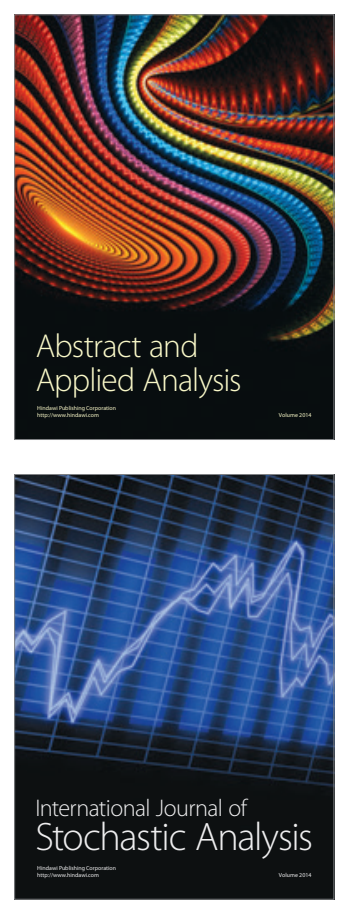

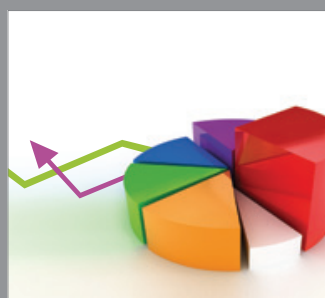

ournal of

Probability and Statistics

Promensencen
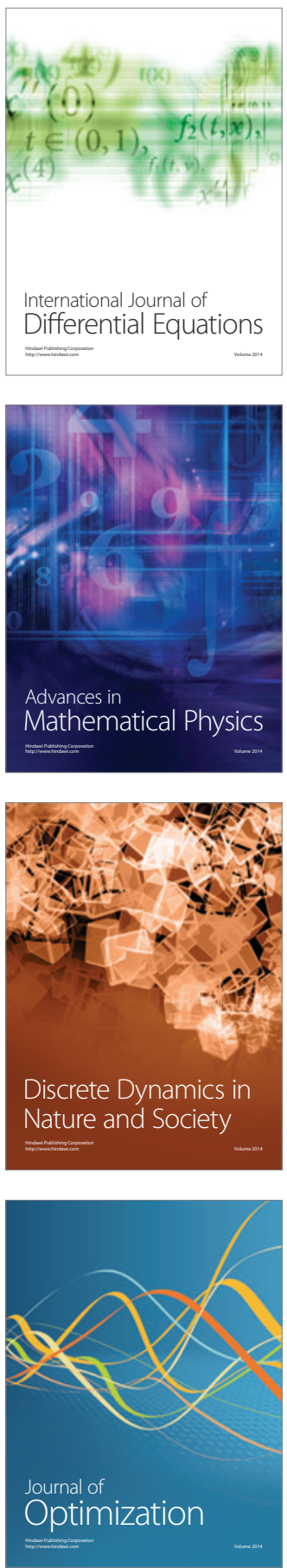